\title{
Incidência de lesão no CrossFit: uma revisão sistemática de literatura
}

\author{
Incidence of injury on CrossFit: a systematic literature review
}

\author{
Dayse Queiroz da Silva Oliveira ${ }^{1}$, Marília Cordeiro Vasconcelos ${ }^{1}$, Ravena Santana Torres ${ }^{1}$, \\ Deisiane Reis Santos ${ }^{1}$, Dario da Silva Monte Nero² \\ ${ }^{1}$ Centro Universitário Leonardo da Vinci (Uniasselvi), Indaial/SC, Brasil \\ ${ }^{2}$ Universidade Federal da Bahia (UFBA), Salvador/BA, Brasil
}

\section{HISTÓRICO DO ARTIGO}

Recebido: 05 junho 2020

Revisado: 17 julho 2020

Aprovado: 31 julho 2020

\section{PALAVRAS-CHAVE:}

Treinamento Intervalado de Alta Intensidade; Exercício Físico;

Ferimentos e Lesões.

\section{KEYWORDS:}

High-Intensity Interval Training; Exercise; Wounds and Injuries.

\section{RESUMO}

INTRODUÇÃO: O CrossFit ${ }^{\circledR}$ é um programa de condicionamento físico que tem como característica movimentos sucessivos englobando uma grande variedade de exercícios que vão desde a corrida aos exercícios de levantamento olímpico e movimentos ginásticos, realizados em alta intensidade com curtos intervalos de recuperação. Nos últimos anos a adesão a esse esporte vem crescendo consideravelmente, e com isso sugerese que os praticantes têm se submetido a treinos pesados e extenuantes que os levam a desenvolver lesões. OBJETIVO: O objetivo deste estudo é realizar uma revisão sistemática para identificar a incidência de lesões no CrossFit e quais são as articulações mais referidos de lesão desta prática.

MÉTODOS: Este estudo consiste em uma revisão bibliográfica realizada nas bases de dados eletrônicas Scielo, Pubmed/Medline com os termos CrossFit e lesão em português e inglês. Foram encontradas no total 64 artigos nas bases de dados pesquisadas, porém apenas 6 artigos foram incluídos.

RESULTADOS: Com base nos estudos analisados os resultados mostram que a incidência de lesão no CrossFit não é alta variando entre 0,27 e 18,9 por 1000 horas de treinamento, sendo maior em indivíduos iniciantes ou indivíduos experientes. Em relação ao local das lesões, ombro, coluna lombar e joelho foram as articulações mais citadas com incidência de lesões, provavelmente por serem as articulações mais solicitadas na maioria dos movimentos.

CONCLUSÃO: Com base nos estudos analisados a incidência de lesão no CrossFit é baixa, e as lesões ocorrem mais frequentemente no ombro, lombar e joelho.

\section{ABSTRACT}

BACKGROUND: CrossFit $^{\circledast}$ is a fitness program that features successive movements encompassing a wide variety of exercises ranging from running to Olympic lifting exercises and gymnastics movements performed in high intensity with short recovery intervals. In recent years, adherence to this sport has grown considerably, and in this way it is suggested that the practitioners have undergone heavy and strenuous workouts that lead them to develop injuries.

OBJECTIVE: The objective of this study is to carry out a systematic review to identify the incidence of injuries in CrossFit and which are the most referred joints of injury in this practice.

METHODS: This study consists of a bibliographic review carried out in the electronic databases Scielo, Pubmed/ Medline with the terms CrossFit and injury in Portuguese and English. A total of 64 articles were found in the databases searched, but only 6 articles were included.

RESULTS: Based on the studies analyzed, the results show that the injury incidence in CrossFit is not high, varying between 0.27 and 18.9 per 1000 hours of training, being higher in beginners or experienced individuals. In relation to the location of the injuries, the shoulder, lumbar spine, and knee were the joints most cited with incidence of injuries, probably because they are the joints most requested in most movements. CONCLUSION: Based on the studies analyzed, the incidence of injury at CrossFit is low, and injuries occur more frequently on the shoulder, lower back, and knee. 


\section{INTRODUÇÃO}

Já é bem conhecido que a prática de exercícios físicos moderados ajuda a melhorar a saúde e aprimorar as capacidades físicas, levando a uma boa condição de saúde e prevenindo doenças. Segundo um posicionamento da Sociedade Brasileira de Medicina do Esporte (1996) as atividades físicas diárias tais como subir escadas e andar podem promover benefícios significativos para a saúde, e somados a programas de exercícios físicos, se tornam ainda mais recomendados. Entre os benefícios promovidos para a saúde estão a melhora do condicionamento físico, da resistência muscular geral e localizada, coordenação motora entre outros (SILVA, 2010). Isso faz com que exercícios combinados ou mesmos treinos como o CrossFit ganhem cava vez mais adeptos.

O CrossFit ${ }^{1}$ é um programa de condicionamento físico que cada vez mais vem ganhando vários adeptos e que tem como característica movimentos sucessivos, abrangentes e generalizados que buscam desenvolver 10 capacidades físicas através de treinos que trabalham movimentos funcionais em alta intensidade, que segundo seu criador, Greg Glassman, gera mais ganhos significativos no condicionamento físico (GLASSMAN, 2005). A sessão de treino segue uma ordem que vai do aquecimento, desenvolvimento de força ou habilidade e a parte do condicionamento (XAVIER; LOPES, 2017). O treino do dia (WOD - workout of the day) são planejados por um coach para que os exercícios sejam realizados o maior número possível dentro de um determinado período de tempo; os treinos são distintos e podem e devem ser adaptadas dependendo das condições dos alunos, e são divididos em etapas: o aquecimento, trabalho de técnica ou força, WOD e alongamento (MOURA et al., 2019).

Segundo Glassman (2005) os treinos podem ser realizados por qualquer pessoa, o que varia é apenas o grau da intensidade e não as necessidades em relação ao ganho de condicionamento físico, não é um treino voltado apenas para atletas, mas é um método testado em várias pessoas com condições distintas. Nesta metodologia de treino existe uma grande variedade de exercícios que vão desde a corrida aos exercícios de levantamento olímpico e movimentos ginásticos, sendo que os exercícios são realizados em alta intensidade e em uma velocidade rápida, com curtos intervalos de recuperação (WEISENTHAL et al., 2014).

De acordo com Xavier e Lopes (2017) por ser uma atividade com alta demanda fisiológica e biomecânica, exigindo do indivíduo o empenho máximo, pode representar um risco de lesões musculoesqueléticas, a uma incapacidade funcional e afastamento das ativididades, sendo lesões entendidas como alterações que levem a um prejuízo das funções musculoesqueléticas e alterações histoquímicas e morfológicas do tecido.

Segundo Bach e Hasan (2003) a lesão é um problema doloroso gerando um impedimento no atleta para que este desempenhe sua prática esportiva, sendo a inflamação um processo comum em todas as lesões, ela é uma resposta tecidual localizada decorrente da lesão, e caracterizado por eventos complexos químicos, vasculares e celulares que levam recuperação e regeneração. Afetam ligamentos, articulações, tendões, discos

${ }^{1}$ NOTA DO EDITOR: CrossFit ${ }^{\oplus}$ é uma marca registrada da Crossfit Inc., sediada nos EUA. Para fins deste estudo, iremos grafar apenas o termo CrossFit, como originalmente foi criado, mas sem o símbolo de marca registrada $\left({ }^{\oplus} \mathrm{ou}^{\mathrm{TM}}\right)$. vertebrais entre outras estruturas que podem ser provocadas ou agravadas pela atividade física (PINHO et al., 2013).

O CrossFit por se caracterizar em uma atividade na qual há uma combinação de vários estilos de exercício físicos, e que tem tomado grandes proporções nos últimos tempos, muitos praticantes acabam se submetendo a treinos muito intensos e extenuantes que tem levado a lesões, e a desistência de alguns praticantes do esporte (LOPES et al., 2018)

Segundo Montalvo et al. (2017) o CrossFit teve sua popularidade aumentada em 2005, e devido a uma maior aceitação da população e a falta de literatura científica que relatasse os efeitos do treinamento, muito tem se questionado sobre sua segurança. Por isto, tem se associado uma alta incidência de lesão à prática do CrossFit devido ao fato de ser uma atividade extremamente intensa, porém não se sabe ao certo a real incidência das lesões e quais são as articulações mais afetadas com esta prática. Portanto, o objetivo do presente estudo consiste em realizar uma revisão sistemática para identificar a incidência de lesões no CrossFit e quais são as articulações mais referidos de lesão nesta modalidade.

\section{MÉTODOS}

Trata-se de uma revisão sistemática seguindo as recomendações PRISMA, realizada nas bases de dados eletrônicas Scielo, Pubmed/Medline, utilizando-se a combinação das palavras-chaves CrossFit AND lesão e CrossFit AND injury. Na base de dados Pubmed foram usadas as palavras-chaves apenas em inglês (CrossFit AND injury), no Scielo em português e inglês (CrossFit AND lesão; CrossFit AND injury), em ambas bases de dados não se utilizou recorte temporal. A busca e seleção dos artigos foram realizadas em junho e julho de 2020.

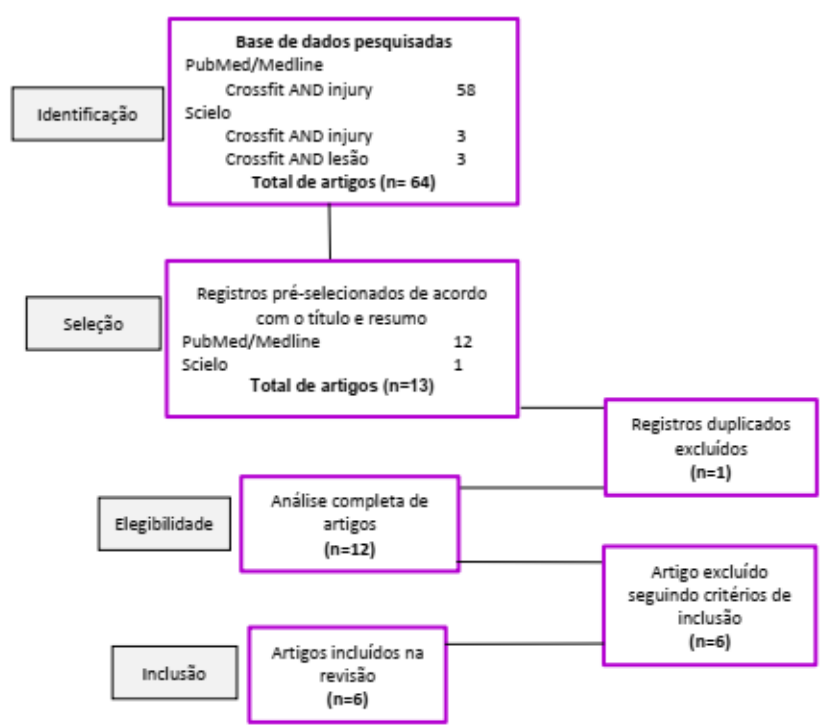

Figura 1. Fluxograma de seleção dos artigos para a revisão.

A seleção dos artigos (Figura 1) foi baseada na leitura do título e resumo e na relevância do mesmo para o objeto de pesquisa deste estudo, os artigos selecionados abordavam especificamente a temática deste estudo. A seleção foi feita por pares 
sem conflito de concordância. Os critérios de inclusão contemplaram estudos que abordassem a incidência de lesão apenas no CrossFit, artigos livres e disponíveis, em ambos os sexos e em qualquer faixa etária, publicados em português e inglês e artigos originais. Os critérios de exclusão foram: artigos de revisão, resultados duplicados (utilizado em outros estudos), outros idiomas e sem resumo disponível. Os artigos que preencheram os critérios de inclusão foram lidos na íntegra. No total 6 artigos foram selecionados e incluídos nesta revisão.

\section{RESULTADOS}

No Quadro 1 é possível visualizar um resumo dos estudos analisados nesta revisão. O estudo de Feito et al. (2018) avaliou incidência de lesão em praticantes de CrossFit durante um período de 4 anos através de um questionário online. Foram incluídos indivíduos com mais de 18 anos e com pelo menos 3 meses de prática no CrossFit. Um total de 3049 indivíduos (1483 mulheres e 1566 homens) responderam ao questionário, deste total, 931 (30,5\%; 495 homens e 436 mulheres) relataram ter tido uma lesão relacionado ao CrossFit, dos quais 581 (62,4\%) tiveram lesão em uma única parte do corpo, e $350(37,6 \%)$ em mais de uma parte.

Os locais mais comuns de lesão foram os ombros (39\%), costas $(36 \%)$, cotovelos $(12 \%)$, joelhos $(15 \%)$ e punho $(11 \%)$, e 6 casos de rabdomiólise $(0,6 \%)$ foram relatados. 0 tempo de experiência no esporte influenciou a taxa de lesão, sendo que indivíduos com mais de 3 anos de experiências tiveram taxas maiores de lesão $(43,1 \%)$, em relação àqueles com 1 a 3 anos $(38,8 \%)$ e menos de 1 ano (18\%), estes resultados podem ser devido a uma maior frequência semanal, intensidade do treinamento e variedade de exercícios, porém o autor não deixa claro essas informações.

Em relação a frequência semanal, indivíduos que praticam de 3 a 5 vezes na semana tiveram mais lesões do que aqueles que faziam menos de 3 vezes ou mais de 5 vezes. As taxas de lesão mostram neste estudo que com base no número máximo de horas semanais treinadas a taxa de lesão foi de 0,27 por 1000 horas de treino (mulheres: 0,28 ; homens: 0,26 ) e com base no número mínimo de horas treinadas por semana a taxa foi de 0,74 por 1000 horas (mulheres: 0,78; homens: 0,70) (FEITO et al., 2018).
Outro estudo realizado com praticantes de CrossFit foi o de Mehrab et al. (2017) através de questionário online. Um total de 449 pessoas ( 226 homens e 183 mulheres) foram incluídas neste estudo, com média de idade de $31,9 \pm 8,3$ anos, sendo que $19,6 \%$ da população $(n=88)$ era composta por indivíduos que tinham menos de 6 meses de experiência no esporte, 21,8\% $(n=98)$ entre 6 e 12 meses, $28,5 \%(n=128)$ entre 12 e 24 meses, e 30,1\% ( $n=135$ ) com mais de 24 meses de experiência. Do tota de questionários avaliados 252 relataram ter tido alguma lesão, sendo que a parte mais afetada foi o ombro $(n=87)$, lombar $(n=48)$ e joelho (25).

Segundo relato dos atletas, as possíveis causas de lesão foram execução incorreta $(n=75)$, fadiga $(n=74)$, carga alta $(n=59)$, desconhecido $(n=37)$, resultado de lesão anterior $(n=34)$ e por causa do treinador $(n=6)$, o relato dos atletas não foi um dado discutido no artigo. Neste estudo a taxa de lesão foi $56,1 \%$ (neste estudo as taxas de lesões não foram calculados por 1000 horas de treinamento).

No estudo de Montalvo et al. (2017) foi avaliada a incidência e fatores de risco para lesão no CrossFit, através de entrevista utilizando um questionário que avaliava os seis meses de treino anteriores em 191 atletas (94 homens e 97 mulheres) através de entrevista realizadas em 4 instalações da CrossFit no sul da Flórida, deste total 50 referiram ter sofrido lesão (total de 62 lesões), sendo que a taxa de incidência de lesão foi 2,3 lesões por 1000 horas de treino. Em relação aos fatores de risco que influenciaram, os mais expressivos foram o tempo de participação no CrossFit, estatura, massa corporal, horas semanais de treino. Com relação à participação na competição, aqueles que declararam competir ( $n=65), 40 \%$ tiveram lesão nos 6 meses anteriores. A prática de outros esportes também estava associada à lesão, de um total de 123 que declaram atividades externas, 31,7\% ( $n=39)$ tiveram lesão em comparação aqueles que fazem exclusivamente o CrossFit $(n=67)$ apenas 10 tiveram lesão (15\%). Os locais que tiveram mais lesões foram o ombro, joelho e lombar. De um total de 62 lesões descritas em partes distintas, 11 eram lesões pré-existentes e 47 se originaram com o CrossFit.

No estudo de Da Costa et al. (2019) foram incluídos atletas e não-atletas das modalidades (estudo prospectivo de 12 meses, os dados foram coletados através de questionário), no total 414

Quadro 1. Estudos avaliados sobre lesão no Crossfit nos periódicos científicos publicados e localizados no PubMed/Medline e Scielo até julho de 2020.

\begin{tabular}{|c|c|c|c|c|c|}
\hline Autores & Amostra & Idade & População avaliada & Tipo de lesão & Incidência \\
\hline Feito et al. (2018) & $\begin{array}{c}3.049 \text { ( } 1.483 \text { mulheres e } \\
1.566 \text { homens })\end{array}$ & $36,8 \pm 9,8$ anos & Praticantes & $\begin{array}{l}\text { Ombro, costas, joelho, } \\
\text { cotovelo, punho e } \\
\text { rabdomiólise }\end{array}$ & $\begin{array}{l}\text { Máximo de horas semanais treinadas= } \\
0,27 / 1000 \text { hrs de treinamento } \\
\text { Mínimo de horas semanais treinadas= } \\
0,74 / 1000 \text { hrs de treinamento }\end{array}$ \\
\hline Mehrab et al. (2017) & $\begin{array}{c}449 \text { (226 homens e } 183 \\
\text { mulheres) }\end{array}$ & $31,9 \pm 8,3$ anos & Atletas & Ombro, lombar e joelho & $56,1 \%$ \\
\hline Montalvo et al. (2017) & $\begin{array}{c}191 \text { (94 homens e } 97 \\
\text { mulheres) }\end{array}$ & $31,69 \pm 9,4$ anos & Atletas & Ombro, joelho e lombar & $2,3 / 1000 \mathrm{hrs}$ de treinamento \\
\hline Da Costa et al. (2019) & $\begin{array}{c}414 \text { (243 homens e } 171 \\
\text { mulheres) }\end{array}$ & $31 \pm 6,6$ anos & $\begin{array}{l}\text { Atletas competitivos, } \\
\text { recreativos e iniciantes }\end{array}$ & Ombro e coluna lombar & $3,24 / 1000 \mathrm{hrs}$ de treinamento \\
\hline Szeles et al. (2020) & $\begin{array}{l}406 \text { (198 homens e } 208 \\
\text { mulheres) }\end{array}$ & $32,1(31,4-32,8)$ anos & Praticantes & Ombro e coluna lombar & $\begin{array}{l}\mathrm{IC}=32,8 \%(95 \% \mathrm{IC}, 28,4-37,5 \%)) \\
\mathrm{DI}=18,9 / 1000 \text { hrs de treinamento }\end{array}$ \\
\hline Larsen et al. (2020) & $\begin{array}{c}168 \text { (51 homens e } 117 \\
\text { mulheres) }\end{array}$ & $29,2 \pm 7,9$ anos & Praticantes & Coluna lombar e joelho & $9,5 / 1000$ horas de treinamento \\
\hline
\end{tabular}

$\mathrm{C}=$ incidência cumulativa

$\mathrm{DI}=$ densidade de incidência 
indivíduos (243 homens e 171 mulheres) com média de idade de 31 anos. Destes 157 (37,9\%) relataram ter sofrido lesão, a incidência de lesão foi 3,24 por 1000 horas de treinamento. Os locais referidos de lesão foram: ombro $(30,8 \%)$, lombar $(30,1 \%)$, perna $(19,2 \%)$, pulso $(14,7 \%)$, joelho $(13,5 \%)$, mão/dedo $(10,9 \%)$, o tornozelo, coxa, braço, coluna cervical, pé, cabeça, abdômen, cotovelo, costela, e antebraço tiveram um percentual abaixo de $10 \%$. Um percentual de $89,1 \%(n=139)$ das lesões foram classificadas como leves a moderadas e $10,9 \%(n=17)$ foram consideradas graves a muito grave.

Neste estudo os fatores de risco para lesão foram lesões anteriores e o tempo de prática no CrossFit. Em atletas competitivos as taxas de lesão foram maiores $(82,2 \%)$ do que em atletas recreativos, com menos experiência. Comparando atletas de nível competitivo com iniciantes, a probabilidade de lesão foi 5 vezes maior, entre atletas de nível recreativo e iniciante foi 2 vezes maior, provavelmente devido a necessidade de superar limites. Estes dados corroboram com o estudo de Feito et al. (2018) no qual a incidência de lesão foi maior em praticantes com tempo maior de experiência do que os iniciantes. Este fato se deve provavelmente a um maior tempo de exposição ao treinamento, necessidade de superar limites e uma melhor proficiência técnica (DA COSTA et al., 2019).

Szeles et al. (2020) investigaram a incidência de lesão e a associação de características pessoais e do treinamento durante 12 semanas em 406 indivíduos (198 homens e 208 mulheres) com média de idade de 32,1 anos. Destes indivíduos, 133 relataram ter sofrido lesão. A incidência cumulativa foi de $32,8 \%$ e a densidade de incidência foi de 18,9 por 1000 horas de treinamento. Os locais mais referidos de lesão foram o ombro $(n=47$, $19 \%)$ coluna lombar $(n=37,15 \%)$ e joelho $(n=29,11,7 \%)$. As cargas de treinamento (Scaled e Rx) e lesões anteriores foram consideradas fatores de risco para lesão, e o aumento de 1 ano de experiência foi considerado um fator protetor. Com relação as cargas de treinamento, o autor sugere que ao sair do Scaled (iniciante) para o Rx (avançado) haverá um aumento na carga de treino, e que deve haver uma atenção maior nesta transição para evitar lesões. Já em relação a experiência ser considerada um fator protetor o autor sugere que mais estudos devam ser feitos.

No estudo de Larsen et al. (2020) investigou a incidência de lesão em indivíduos iniciantes no CrossFit, através de questionário (autorrelato), durante um período de treinamento de 8 semanas que só incluiu indivíduos que nunca haviam praticado a modalidade. Foram incluídos 168 indivíduos (51 homens e 117 mulheres) com média de idade de $29,2 \pm 7,9$ anos. Os resultados mostram que 22 indivíduos relataram 1 lesão $(13,1 \%)$ e 3 relataram 2 lesões (1,8\%), totalizando 28 lesões em 25 indivíduos. A incidência de lesão foi de 9,5 por 1000 horas de treinamento. As regiões referidas de lesão foram: a coluna lombar $(n=7,25 \%)$, joelho ( $n=6,21,4 \%)$, cotovelo/mão $(n=5,17,9 \%)$, "outras localizações anatômicas" ( $n=5,17,9 \%)$, ombro $(n=2,7,1 \%)$, pescoço $(n=1,3,6 \%)$, quadril $(n=1,3,6 \%)$ e tornozelo $(n=1,3,6 \%)$.

De acordo com os estudo analisados há um risco maior em indivíduos iniciantes, que se deve ao fato de não passarem por um período de adaptação ao treinamento e aprendizagem das técnicas e execuções corretas, outras já referem maior incidência em indivíduos experientes, que provavelmente se deve ao fato de treinarem com altos volumes e cargas intensas, além de uma maior frequência semanal sem período adequado de recuperação. Segundo o Mehrab (2017), o curto tempo de participação na modalidade tem contribuído para a ocorrência das lesões, menos de seis meses na modalidade, isso associado a treinos excessivos e a complexidade dos exercícios e movimentos, este fato pode ser reduzido com uma maior atenção a participantes iniciantes e na redução do volume e intensidade dos treinos, todos estes dados são uteis como base para a realização de futuras pesquisas na prevenção de lesões no CrossFit. (MEHRAB et al., 2017).

Em relação a localização, nos estudos analisados as articulações mais referidas de lesão foram a do punho, coluna lombar e ombro, segundo Lopes et al. (2018) estas lesões estão associadas a alta quantidade de peso e repetições típica do esporte. Vale ressaltar, que alguns dos estudos analisados (SZELES et al., 2020; FEITO et al., 2018; LARSEN et al., 2020; MEHRAB et al., 2017) foram auto-relatados e não tiveram a orientação de um profissional sobre lesões, apenas uma descrição e/ou imagem nos questionários, o que pode prejudicar não somente na identificação exata da lesão, pela falta de validação médica, deixando livre para interpretação pessoal além do viés de memória.

\section{CONCLUSÃO}

Com base nos estudos analisados a incidência de lesão no CrossFit não é alta, sendo semelhante em alguns estudos e em outros um pouco mais elevada, provavelmente devido a característica da população (atletas competitivos, recreativos e iniciantes) e a definição de lesão adotada pelos estudos. Em relação à localização das lesões, ombro, lombar e joelho foram as articulações mais citadas com incidência de lesão, isso se deve ao fato de serem as articulações mais solicitadas na maioria dos movimentos do esporte, sendo bastante associado aos movimentos ginásticos e levantamento de peso.

Os estudos apresentam limitações em relação a lesões auto referidas, podendo levar a um erro de interpretação, apesar de alguns estudos terem definido o que seria lesão e/ou imagens ao aplicar o questionário, mas o viés da memória também é importante porque ao fazer uma análise retrospectiva os participantes poderiam ter se esquecido de alguma eventualidade que tiveram em decorrência do CrossFit, outra limitação foi a definição de lesão variou entre os estudos.

\section{REFERÊNCIAS}

BACH, B. R.; HASAN, S. S. Lesões esportivas e a resposta dos tecidos à lesão física. In: SCHENCK R. C. J. Medicina esportiva e treinamento atlético. 3. ed. São Paulo: Roca. 2003. p. 128.

DA COSTA, T. S.; LOUZADA, C. T. N.; MIYASHITA, G. K.; SILVA, P. H. J. da; SUNGAILA, H. Y. F.; LARA, P. H. S.; ... ; ARLIANI, G. C. CrossFit: injury prevalence and main risk factors. Clinics, São Paulo, v. 74, p. e1402, 2019.

FEITO, Y.; BURROWS, E. K.; TABB, L. P. A 4-year analysis of the incidence of injuries among crossfit-trained participants. Orthopaedic Journal of Sports Medicine, Thousand Oaks, v. 6, n. 10, p. 2325967118803100, 2018.

GLASSMAN, G. Guia de treinamento. CrossFit Journal, Whashington, 2005. Coletânea de artigos publicados no CrossFit Journal, disponível em: <http://library.crossfit.com/free/pdf/CFJ L1 TG Portuguese.pdf>. Acessado em: 31 de março de 2019.

LARSEN, R. T.; HESSNER, A. L.; ISHOI, L.; LANGBERG, H.; CHRISTENSEN, J. Injuries in novice participants during an eight-week start up Crossfit program: 
a prospective cohort study. Sports, Basel, v. 8, n. 2, p. 21 (1-12), 2020.

LOPES, P.; BEZERRA, F. H. G.; NADSON FILHO, A.; BRASILEIRO, I.; PACHECO NETO, P.; SANTOS JUNIOR. Lesões osteomioarticulares entre os praticantes de crossfit. Motricidade, Ribeira de Pena, v. 14, n. 1, p. 266-70, 2018.

MONTALVO, A. M.; SHAEFER, H., RODRIGUEZ, B.; LI, T.; EPNERE, K.; MYER, G. $D$. Retrospective injury epidemiology and risk factors for injury in CrossFit. Journal of Sports Science Medicine, Bursa, v. 16, n. 1, p. 53-9, 2017.

MOURA, D. L.; FROHN, I.; TORRES, J. P.; INÊS, A.; FONSECA, F. Biomecânica e traumatologia no CrossFit. Revista Medicina Desportiva Informa, Lisboa, v. 10, n. 2, p. 15-7, 2019.

PINHO, M. C.; VAZ, M. P.; AREZES, P. M.; CAMPOS, J. R.; MAGALHÃES, A. B. Lesões músculo-esqueléticas relacionadas com as atividades desportivas em crianças e adolescentes: uma revisão das questões emergentes. Motricidade, Vila Real, v. 9, n. 1, p. 31-49, 2013.

SILVA, O. C. A qualidade de vida ligada à prática regular de atividades físicas. Lecturas: Educación Física y Deporte, Revista Digital, Buenos Aires, n. 150, 2010. Disponível em: <https://www.efdeportes.com/efd150/pratica-regular-de-atividades-fisicas.htm>. Acessado em: 16 de julho de 2020.

SBME. Sociedade Brasileira de Medicina do Exercício e do Esporte. Posição oficial da Sociedade Brasileira de Medicina do Esporte: atividade física e saúde. Revista Brasileira de Medicina do Esporte, São Paulo, v. 2, n. 4, p. 79-81, 1996.

SZELES, P. R. Q.; COSTA, T. S. da; CUNHA, R. A.; HESPANHOL, L.; POCHINI, A. C.; RAMOS, L. A.; COHEN, M. Crossfit and the epidemiology of musculoskeletal injuries: a prospective 12-week cohort study. Orthopaedic Journal of Sports Medicine, Thousand Oaks, v. 8, n. 3, p. 2325967120908884, 2020.

WEISENTHAL, B. M.; BECK, C. A.; MALONEY, M. D.; DEHAVEN, K. E.; GIORDANO, B. D. Injury rate and patterns among crossfit athletes. Orthopaedic Journal of Sports Medicine, Thousand Oaks, v. 2, n. 4, p. 1-7, 2014.

XAVIER, A. A.; LOPES, A. M. C. Lesões musculoesqueléticas em praticantes de Crossfit. Revista Interdisciplinar Ciências Médicas, Belo horizonte, v. 1, n. 1, p. 11-27, 2017.

\section{CONFLITO DE INTERESSE}

Os autores do estudo declaram não haver conflito de interesses.

\section{FINANCIAMENTO}

Este estudo não teve apoio financeiro.

\section{ORCID E E-MAIL DOS AUTORES}

Dayse Queiroz da Silva Oliveira (Autor Correspondente) ORCID: 0000-0001-9783-229X.

E-mail: dayse_queiroz@live.com

Marília Cordeiro Vasconcelos

ORCID: 0000-0001-7757-2558.

E-mail: maraedf@icloud.com

Ravena Santana Torres

ORCID: 0000-0003-2706-3557.

E-mail: ravenauefs@hotmail.com

Deisiane Reis Santos

ORCID: 0000-0003-1056-9631.

E-mail: deisyrs71@gmail.com

Dario da Silva Monte Nero

ORCID: 0000-0001-5092-5149.

E-mail: dariomontenero@yahoo.com.br 